\title{
Influence of Heat Transfer on MHD Oscillatory Flow for Williamson Fluid with Variable Viscosity Through a Porous Medium
}

\author{
Wissam Sadiq Khudair, Dheia Gaze Salih Al-Khafajy \\ Faculty of Computer Science and Information Technology, University of Al-Qadisiyah, Diwaneyah, Iraq \\ Email address: \\ wissamhsse12@gmail.com(W.S.Khudair),dheia.salih@qu.edu.iq(D.G.S.Al-Khafajy) \\ *Corresponding author
}

To cite this article:

Wissam Sadiq Khudair, Dheia Gaze Salih Al-Khafajy. Influence of Heat Transfer on MHD Oscillatory Flow for Williamson Fluid with Variable Viscosity Through a Porous Medium. International Journal of Fluid Mechanics \& Thermal Sciences. Vol. 4, No. 1, 2018, pp. 11-17. doi: $10.11648 /$ j.ijfmts.20180401.12

Received: February 21, 2018; Accepted: March 9, 2018; Published: April 3, 2018

\begin{abstract}
The main theme of the present examined the influence of heat transfer on magnetohydrodynamics (MHD) for the oscillatory flow of Williamson fluid with variable viscosity model for two kinds of geometries "Poiseuille flow and Couette flow" through a porous medium channel. The momentum equation for the problem, is a non-linear differential equations, has been found by using "perturbation technique" and intend to calculate the solution for the small number of Weissenberg (We $<<1$ ) to get clear forms for the velocity field by assisting the (MATHEMATICA) program to obtain the numerical results and illustrations. The physical features of Darcy number, Reynolds number, Peclet number, magnetic parameter, Grashof number and radiation parameter are discussed simultaneously through presenting graphical discussion. Investigated through graphs the variation of a velocity profile for various pertinent parameters. While the velocity behaves strangely under the influence of the Brownian motion parameter and local nanoparticle Grashof number effect. On the basis of this study, it is found that the velocity directly with Grashof number, Darcy number, radiation parameter, Reynolds number and Peclet number, and reverse variation with magnetic parameter and frequency of the oscillation and discussed the solving problems through graphs.
\end{abstract}

Keywords: Williamson Fluid, Variable Viscosity, Heat Transfer, (MHD), Porous Medium

\section{Introduction}

Central porosity is a matter containing a number of small holes distributed throughout the matter. A porous medium flows through the fluid infiltration and water infiltration into the river beds. The movement of groundwater, water, and oils are some important examples of flows through porous means. The oil tank often contains a sedimentary structure such as limestone and sandstone in which the oil is contained. Another example of flow through a porous medium is leakage under the dam which is very important. Examples: of natural porosity such as sand ash, wood, filtering, human lung, bitterness and yellow stones, oil production engineering and many other processes.

In [1] show the exact solutions for fourth kinds of flows between two parallel plates. [2] studied the influence of inclined magnetic field between two infinite parallel plates, [3] discussed the laminar flow between parallel plates under the action of the transverse magnetic field and heat transfer. [4] discussed the two kinds of geometries Poiseuille flow and Couette flow of Carreau fluid with pressure dependent viscosity in a variable porous medium. [5] examined the flow of Williamson fluid for two kinds of geometries Poiseuille flow and Couette flow in an inclined channel.

Viscosity is one of the most important specifications for fluids, [6] studied the variable viscosity through a porous medium and used the homotopy analysis method to solve the problem. [7] studied the related of the variable viscosity through a porous medium by using generalized Darcy's law, to solve the problem he using the perturbation technique. [8] Influence of heat transfer on magneto hydrodynamics 
oscillatory flow for Williamson fluid through a porous medium. [9] studied the variable viscosity of Jeffrey fluid in an asymmetric channel.

Consider a mathematical model to influence of heat transfer on MHD oscillatory flow for Williamson fluid with variable viscosity through a porous medium channel. The perturbation technique series use to solve the problem for the two kinds of flow Poiseuille flow and Couette flow are addressed. The result of the physical parameters problem was discussed by using the graphs.

\section{Mathematical Formulation}

Consider the flow of a Williamson fluid in the channel of breadth $l$ qualify the effects of magnetic field and radioactive heat transference as described in Figure 1. Supposed that the fluid has very small electromagnetic force produced and the electrical conductivity is small. Considering a Cartesian coordinate system such that, $(v(y), 0,0)$ is the velocity vector in which $v$ is the $x$-component of velocity and $y$ is orthogonal to the $x$-axis.

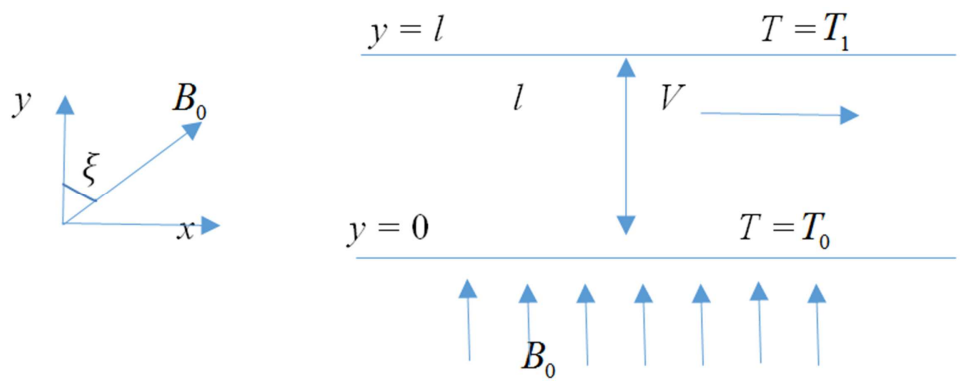

Figure 1. Physical model. [10]:

The constitutive equation for Williamson fluid is given by

$$
\begin{gathered}
\boldsymbol{S}=-\bar{p} \boldsymbol{I}+\tau \\
\bar{\tau}=\left[\mu_{\infty}+\left(\mu_{0}-\mu_{\infty}\right)(1+\Gamma \bar{\gamma})^{-1}\right] A^{*} \\
\dot{\gamma}=\sqrt{\frac{1}{2} \sum_{i} \sum_{j} \dot{\gamma}_{i j} \dot{\gamma}_{j i}}=\sqrt{\frac{1}{2} \Pi \text { and } \prod=\operatorname{tr}\left(\mathrm{A}^{*}\right)^{2}, \mathrm{~A}^{*}=\Delta \bar{V}+(\Delta \bar{V})^{T}}
\end{gathered}
$$

where $\bar{p}$ is the pressure, $\bar{\tau}$ is the extra stress tensor, $\Gamma$ is the time constant, $I$ is the unit tensor, $\mu_{\infty}$ is the infinite shear rate viscosity and $\mu_{0}=\mu(T)$ then $\dot{\gamma}$ is given by:

where $(\nabla \bar{V})$ Is the fluid velocity in the Cartesian coordinates $(x, y, z), \mu_{\infty}=0$ and $\Gamma \dot{\gamma}<1$. Then we can be writing the constitutive equation for Williamson fluid extra stress tensor by follows as:

$$
\bar{\tau}=\mu(T)[(1+\Gamma \bar{\gamma})] A^{*}
$$

The equations of momentum and energy governing such a flow, subjugate to the Boussinesq approximation, are:

$$
\rho \frac{\partial \bar{v}}{\partial \bar{t}}=-\frac{\partial \bar{p}}{\partial \bar{x}}+\frac{\partial \bar{\tau}_{\overline{x x}}}{\partial \bar{x}}+\frac{\partial \bar{\tau}_{\overline{x y}}}{\partial \bar{y}}+\frac{\partial \bar{\tau}_{\overline{x z}}}{\partial \bar{z}}+\rho g \beta\left(T-T_{0}\right)-\sigma B_{0}^{2} \operatorname{Sin}^{2}(\xi) \bar{v}-\frac{\mu(T)}{k} \bar{v}
$$

$$
\rho \frac{\partial T}{\partial \bar{t}}=\frac{K}{C_{p}} \frac{\partial^{2} T}{\partial \bar{y}^{2}}-\frac{1}{C_{p}} \frac{\partial q}{\partial y}
$$

The temperatures at the walls of the channel are given as:

$$
T=T_{0} \text { at } \bar{y}=0 \text {, and } T=T_{1} \text { at } \bar{y}=l .
$$

where $\bar{v}$ is the axial velocity, $K$ is a thermal conductivity, $T$ is a fluid temperature, $\rho$ is a fluid density, $\beta$ is a coefficient of volume amplification due to temperature, $g$ is a hastening due to gravity, $k$ is a permeability, $c_{p}$ is a specific heat at constant pressure, $\sigma$ is a conductivity of the fluid, $\mu(T)$ fluid

$$
\left.\begin{array}{c}
v=\frac{\bar{v}}{V}, x=\frac{\bar{x}}{l}, y=\frac{\bar{y}}{l}, \theta=\frac{T-T_{0}}{T_{1}-T_{0}}, t=\frac{\bar{t} V}{l}, p=\frac{\bar{p} h}{\mu V}, M^{2}=\frac{\sigma B_{0}^{2} h^{2}}{\mu} \\
\mu(\theta)=\frac{\mu(T)}{\mu_{0}}, R e=\frac{\rho l V}{\mu}, P e=\frac{\rho l V c_{p}}{K}, N^{2}=\frac{4 \eta^{2} l^{2}}{K}, \tau_{x x}=\frac{l}{\mu_{0} V} \bar{\tau}_{\overline{x x}} \\
D a=\frac{k}{l^{2}}, G r=\frac{\rho g \beta l^{2}\left(T-T_{0}\right)}{\mu V}, \dot{\gamma}=\frac{l}{V} \bar{\gamma}, \tau_{x y}=\frac{l}{\mu_{0} V} \bar{\tau}_{\overline{x y}}, \tau_{x z}=\frac{l}{\mu_{0} V} \bar{\tau}_{\overline{x z}}
\end{array}\right\}
$$

where $V$ is the mean flow velocity, Darcy number $D a$, Reynolds number Re, Peclet number $P e$, magnetic parameter $M$, 
Grashof number $G r$ and radiation parameter $N$.

Substituting (8) and (9) into (5) - (6), we obtain:

$$
\begin{gathered}
\rho \frac{V \partial v}{\frac{l}{V} \partial t}=-\frac{\frac{\mu_{0} V}{l} \partial p}{l \partial x}+\frac{\frac{\mu_{0} V}{l} \partial \tau_{x x}}{l \partial x}+\frac{\frac{\mu_{0} V}{l} \partial \tau_{x y}}{l \partial y}+\frac{\frac{\mu_{0} V}{l} \partial \tau_{x z}}{l \partial z}+\rho g \beta\left(T_{1}-T_{0}\right) \theta-\sigma B_{0}^{2} \operatorname{Sin}^{2}(\xi) V v-\frac{\mu(\theta) \mu_{0} V}{k} v \\
\rho \frac{\left.\partial\left(\theta\left(T_{1}-T_{0}\right)+T_{0}\right)\right)}{\frac{l}{V} \partial t}=\frac{k}{C_{P}}\left[\frac{\left.\partial^{2}\left(\theta\left(T_{1}-T_{0}\right)+T_{0}\right)\right)}{l^{2} \partial y^{2}}-\frac{1}{k} 4 \eta^{2}\left(T_{0}-T\right)\right]
\end{gathered}
$$

where $\tau_{x x}=0, \tau_{x y}=\mu(\theta)\left[\left(1+\Gamma \frac{\partial v}{\partial y}\right)\right] \frac{\partial v}{\partial y}, \tau_{x z}=0$.

The non-dimensional boundary conditions for temperature equation:

$$
\theta(0)=0, \theta(1)=1
$$

Finally, the non-dimensional equations are:

$$
\begin{aligned}
& R e \frac{\partial v}{\partial t}=-\frac{\partial p}{\partial x}+\frac{\partial}{\partial y}\left[\mu(\theta)\left(\frac{\partial v}{\partial y}+W e\left(\frac{\partial v}{\partial y}\right)^{2}\right)\right]+G r \theta_{0}-\left(M_{1}^{2}+\frac{\mu(\theta)}{D a}\right) v \\
& \rho \frac{\partial \theta}{\partial t}=\frac{\partial^{2} \theta}{\partial y^{2}}+N^{2} \theta \\
& \mu(\theta)=e^{-\alpha \theta}
\end{aligned}
$$

where $M_{1}=M \operatorname{Sin}^{2}(\xi), M$ is the Hartmann number.

To solve the temperature (14) with boundary conditions (12), let

$$
\theta(y, t)=\theta_{0}(y, t) e^{i \omega t}
$$

The frequency of the oscillation denoted by $\omega$.

Substituting the (15) into the (14)

$$
\frac{\partial^{2} \theta}{\partial y^{2}}+\left(N^{2}-i \omega P e\right) \theta_{0}=0
$$

The solution of (16) with boundary conditions (12) is $\theta_{0}(y)=\csc (\varphi) \sin (\varphi)$, where $\varphi=\sqrt{N^{2}-i \omega P e}$. Therefore

$$
\theta(y, t)=\csc (\varphi) \sin (\varphi) e^{i \omega t}
$$

\section{Solution of the Problem}

Now, we solve (13) for two kinds of geometries "Poiseuille flow and Couette flow".

(i) Poiseuille flow

Suppose that the rigid flakes at $y=0$ and $y=l$ are at rest. Therefore

$$
\bar{v}=0 \text { at } \bar{y}=0 \text {, and } \bar{v}=0 \text { at } \bar{y}=l
$$

The non-dimensional boundary conditions:

$$
v(0)=0, v(1)=0 \text {. }
$$

To solve the momentum (13), let

$$
\begin{gathered}
-\frac{\partial p}{\partial x}=\lambda e^{i \omega t} \\
v(y, t)=v_{0}(y, t) e^{i \omega t}
\end{gathered}
$$

Where $\lambda$ is a real constant.

The "Reynold's model" given by variation of viscosity with temperature defined as:
By using the Maclaurin series, we get:

$$
\mu(\theta)=1-\alpha \theta \alpha<<1
$$

In a case when the viscosity is fixed here $\alpha=0$. Substituting (22) into (13), we get:

$$
\begin{gathered}
\operatorname{Re} \frac{\partial v}{\partial t}=-\frac{\partial p}{\partial x}+\frac{\partial}{\partial y}\left[(1-\alpha \theta)\left(\frac{\partial v}{\partial y}+W e\left(\frac{\partial v}{\partial y}\right)^{2}\right)\right]+\operatorname{Gr} \theta_{0}- \\
\left(M_{1}^{2}+\frac{(1-\alpha \theta)}{D a}\right) v
\end{gathered}
$$

Equation (23) is non-linear differential equations and it is hard to get an exact solution, so by use the perturbation technique to find the problem solution, we write:

$$
v_{0}=v_{00}+W e v_{01}+W e^{2} v_{02}+0\left(W e^{3}\right)
$$

Substituting (24) into (23) with boundary conditions (18), then we equality the powers of $(W e)$ :

$A$ - Zeros-order system $\left(W e^{0}\right)$

$$
(1-\alpha \theta) \frac{\partial v_{00}}{\partial y^{2}}-\left(M_{1}^{2}+\operatorname{Rei\omega }+\frac{(1-\alpha \theta)}{D a}\right) v_{00}=-\left(\lambda+\operatorname{Gr} \theta_{0}\right)
$$

The associated boundary conditions are:

$$
v_{00}(0)=v_{00}(1)=0
$$

$B$ - First-order system $\left(W e^{1}\right)$

$$
\begin{gathered}
(1-\alpha \theta) \frac{\partial v_{01}}{\partial y^{2}}-\left(M_{1}^{2}+R e i \omega+\frac{(1-\alpha \theta)}{D a}\right) v_{01}= \\
-2\left(\frac{\partial v_{00}}{\partial y} \frac{\partial^{2} v_{00}}{\partial y^{2}}\right) e^{i \omega t}
\end{gathered}
$$

The associated boundary conditions are:

$$
v_{01}(0)=v_{01}(1)=0
$$

$C$ - Second-order system $\left(W e^{2}\right)$

$$
\begin{gathered}
(1-\alpha \theta) \frac{\partial v_{02}}{\partial y^{2}}-\left(M_{1}^{2}+\operatorname{Rei\omega }+\frac{(1-\alpha \theta)}{D a}\right) v_{02}= \\
-2\left(\frac{\partial v_{00}}{\partial y} \frac{\partial^{2} v_{01}}{\partial y^{2}}+\frac{\partial v_{01}}{\partial y} \frac{\partial^{2} v_{00}}{\partial y^{2}}\right) e^{i \omega t}
\end{gathered}
$$


The associated boundary conditions are:

$$
v_{02}(0)=v_{02}(1)=0
$$

Giving some physical meaning to the problem by considering that when $\alpha$ is small and by using the perturbationseries with parameters $\alpha$. Substitute for $v_{i}$ (For $i$

$$
\begin{gathered}
(1-\alpha \theta) \frac{\partial}{\partial y^{2}}\left(v_{00}+\alpha v_{01}+\alpha^{2} v_{02}\right)-\left(M_{1}^{2}+\operatorname{Rei\omega }+\frac{(1-\alpha \theta)}{D a}\right)\left(v_{00}+\alpha v_{01}+\alpha^{2} v_{02}\right)=-\left(\lambda+\operatorname{Gr} \theta_{0}\right) \\
(1-\alpha \theta) \frac{\partial}{\partial y^{2}}\left(v_{10}+\alpha v_{11}+\alpha^{2} v_{12}\right)-\left(M_{1}^{2}+\operatorname{Rei\omega }+\frac{(1-\alpha \theta)}{D a}\right)\left(v_{10}+\alpha v_{11}+\alpha^{2} v_{12}\right)= \\
-2\left[\frac{\partial}{\partial y}\left(v_{00}+\alpha v_{01}+\alpha^{2} v_{02}\right) \frac{\partial^{2}}{\partial y^{2}}\left(v_{00}+\alpha v_{01}+\alpha^{2} v_{02}\right)\right] e^{i \omega t} \\
(1-\alpha \theta) \frac{\partial}{\partial y^{2}}\left(v_{20}+\alpha v_{21}+\alpha^{2} v_{22}\right)-\left(M_{1}^{2}+\operatorname{Rei\omega }+\frac{(1-\alpha \theta)}{D a}\right)\left(v_{20}+\alpha v_{21}+\alpha^{2} v_{22}\right)= \\
-2\left[\frac{\partial}{\partial y}\left(v_{00}+\alpha v_{01}+\alpha^{2} v_{02}\right) \frac{\partial^{2}}{\partial y^{2}}\left(v_{10}+\alpha v_{11}+\alpha^{2} v_{12}\right)+\frac{\partial}{\partial y}\left(v_{10}+\alpha v_{11}+\alpha^{2} v_{12}\right) \frac{\partial^{2}}{\partial y^{2}}\left(v_{00}+\alpha v_{01}+\alpha^{2} v_{02}\right)\right] e^{i \omega t}(34)
\end{gathered}
$$

The solutions of (32) - (34), are a very long. Finally, the perturbation solutions up to a second term for $v$ is given by:

$$
v=\left(v_{00}+\alpha v_{01}+\alpha^{2} v_{02}\right)+W e\left(v_{10}+\alpha v_{11}+\alpha^{2} v_{12}\right)+W e^{2}\left(v_{20}+\alpha v_{21}+\alpha^{2} v_{22}\right)
$$

\section{(ii) Couette flow}

The upper flake is locomotion and the lower flake is fixed with the velocity $V_{h}$. The boundary conditions for the Couette flow problem as defined:

$$
v(0)=0, v(1)=V_{0}
$$

By the same governing equation in Poiseuille flow (13). The solution, in this case, has been calculated by the perturbation technique and the results have been discussed through graphs.

\section{Results and Discussion}

We discussed the influence of heat transfer on MHD oscillatory flow for Williamson fluid with variable viscosity through a porous medium for " Poiseuille flow and Couette flow" in some results through the graphical illustrations. The analytical results and some of the graphical significant results are presented in figures 2-20. The momentum equation is resolved by using perturbation technique [12] and all the results are discussed graphically by assisting the (MATHEMATICA) program.

The velocity profile of Poiseuille flow is shown in figures 2-9. Figure 2 shows the velocity profile $v$ decreases by the increasing frequency of the oscillation parameter $\omega$. Figure 3 illustrates the influence Grashof number $\mathrm{Gr}$ on the velocity profiles function $v$ vs. $y$, it is found by the increasing $G r$ the velocity profiles function $v$ increases. Figure 4 shows that velocity profile $v$ rising up by the increasing influence the parameter $\lambda$.

Velocity profiles increase by the increasing of Darcy number in Figure 5. Figure 6 illustrates the influence magnetic parameter $M$ on the velocity profiles function $v$ vs. $y$, it is found by the increasing $M$ the velocity profiles function $v$ decreases. Figure 7 shows the velocity profile $v$ rising up by the increasing radiation parameter $N$. Figure 8 show that velocity profile $v$ increases by the increasing influence the Reynolds number. Figure 9 shows the velocity profile $v$ decreases with the increasing $\xi$.
The velocity profile of Couette flow is shown in figures 10-17. We found that by the increasing each of parameters $\operatorname{Re}, G r, D a, N$ and $\lambda$ the velocity profile $v$ increases, while $v$ decreases by the increasing $M, \xi$ and $\omega$.

Based on (17), Figure 18 show that influence of $N$ on the temperature function $\theta$. The temperature increases by the increase in $N$. Figure 19 observed that the influence $P e$ in temperature $\theta$ by the increasing $P e$ then $\theta$ increases. Figure 20 show us that with the increasing of $\omega$ the temperature $\theta$ decreases.

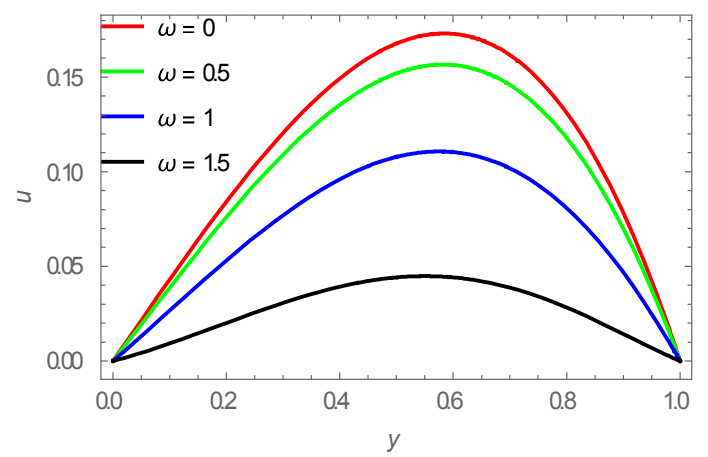

Figure 2. "Poiseuille flow" Velocity profile for $\omega$ with $M=1, N=1, G r=$ $1, D a=0.8, R e=1, P e=1, \lambda=1, W e=0.05, \xi=\frac{\pi}{4}, \alpha=0.02, t=0.5$.

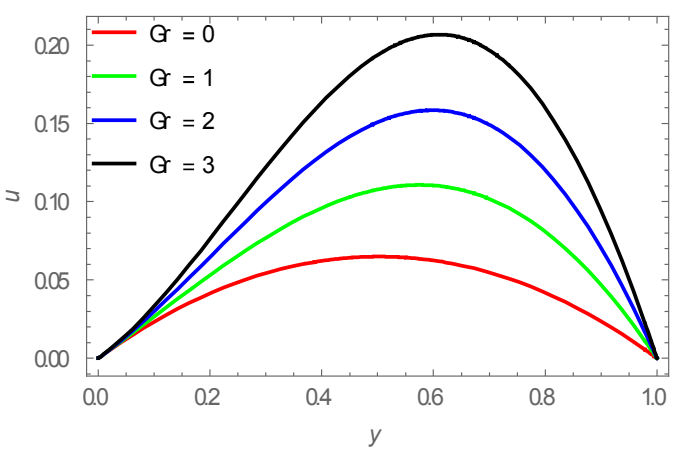

Figure 3. "Poiseuille flow," Velocity profile for Gr with $M=1, N=1, \omega=$ $1, D a=0.8, R e=1, P e=1, \lambda=1, W e=0.05, \xi=\frac{\pi}{4}, \alpha=0.02, t=0.5$. 


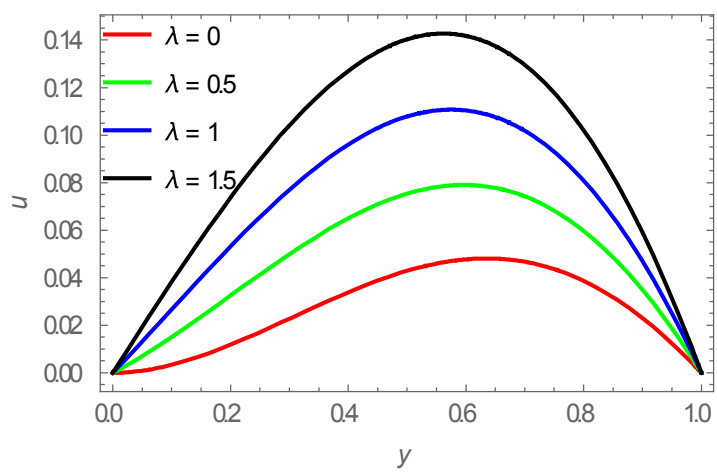

Figure 4. "Poisenille flow," Velocity profile for $\lambda$ with $M=1, N=1, \omega=$ $1, D a=0.8, R e=1, P e=1, G r=1, W e=0.05, \xi=\frac{\pi}{4}, \alpha=0.02, t=0.5$.

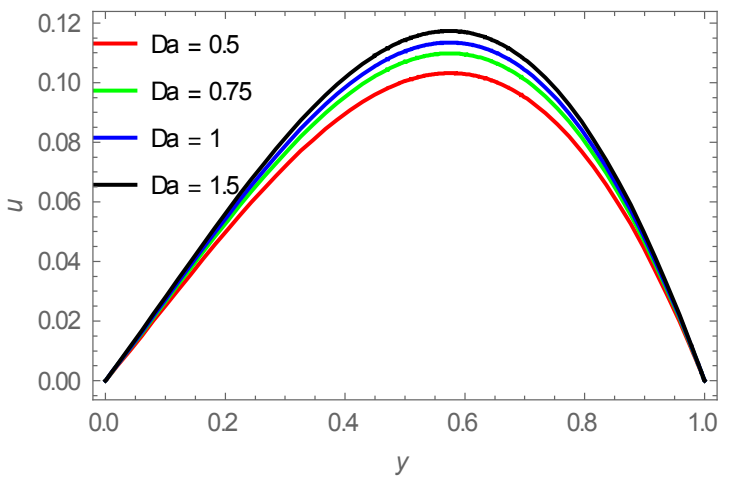

Figure 5. "Poiseuille flow," Velocity profile for Da with $M=1, N=1, \omega=$ $1, \lambda=1, R e=1, P e=1, G r=1, W e=0.05, \xi=\frac{\pi}{4}, \alpha=0.02, t=0.5$.

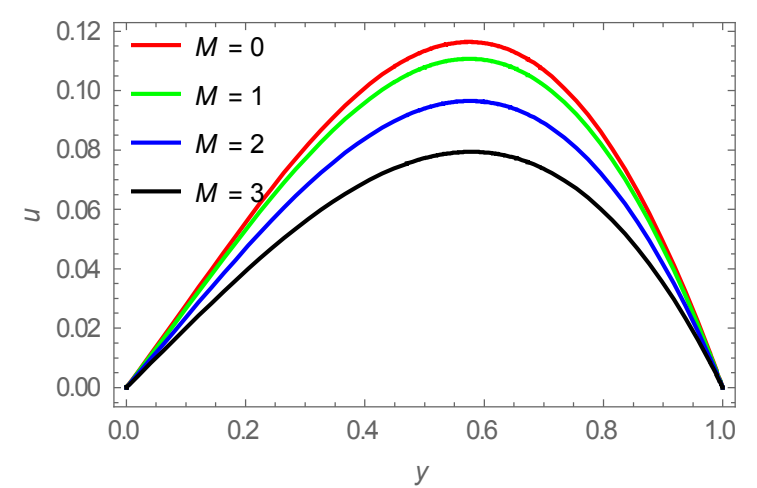

Figure 6. 'Poisenille flow,' Velocity profile for $M$ with $D a=0.8, N=1, \omega=$ $1, \lambda=1, R e=1, P e=1, G r=1, W e=0.05, \xi=\frac{\pi}{4}, \alpha=0.02, t=0.5$.

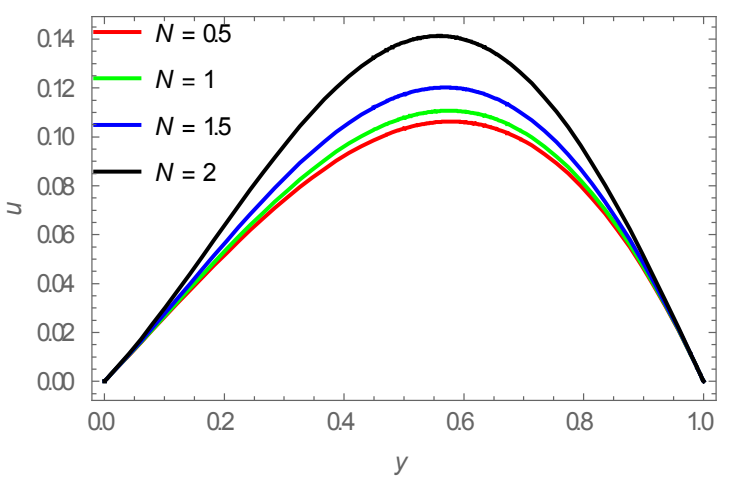

Figure 7. 'Poiseuille flow,' Velocity profile for $N$ with $D a=0.8, M=1, \omega=$ $1, \lambda=1, R e=1, P e=1, G r=1, W e=0.05, \xi=\frac{\pi}{4}, \alpha=0.02, t=0.5$.

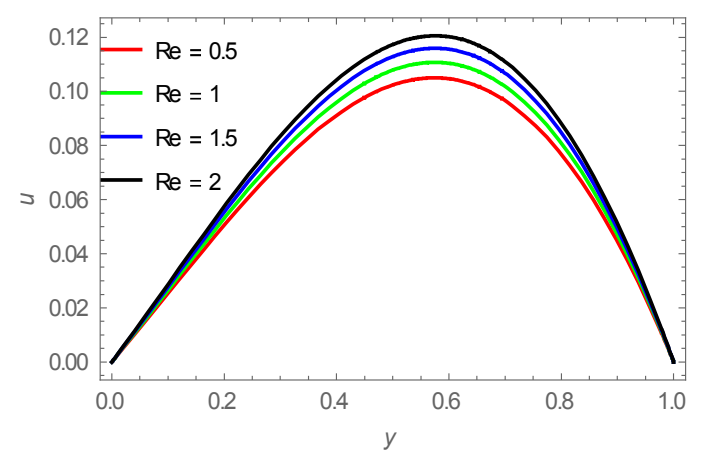

Figure 8. "Poisenille flow," Velocity profile for Re with $D a=0.8, M=$ $1, \omega=1, \lambda=1, P e=1, N=1, G r=1, W e=0.05, \xi=\frac{\pi}{4}, \alpha=0.02, t=$ 0.5 .

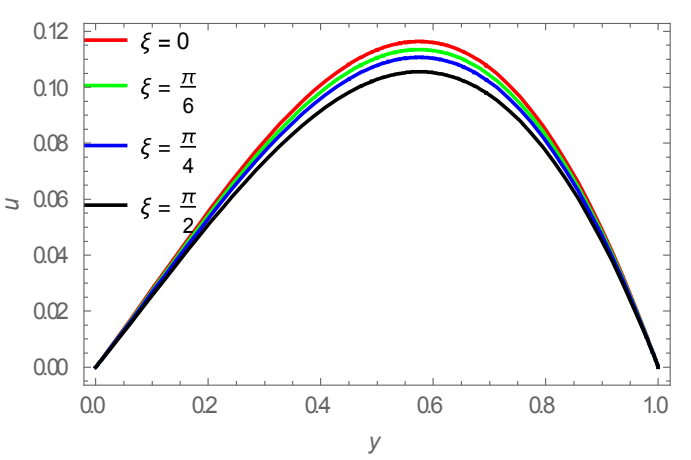

Figure 9. "Poiseuille flow," Velocity profile for $\xi$ with $D a=0.8, M=1, \omega=$ $1, \lambda=1, P e=1, N=1, G r=1, W e=0.05, R e=1, \alpha=0.02, t=0.5$.

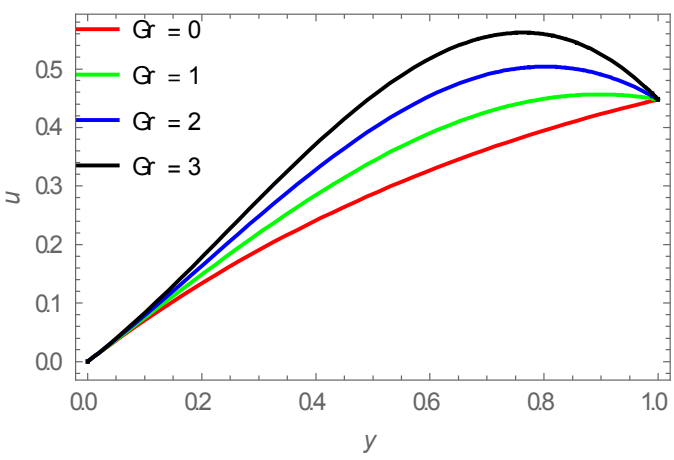

Figure 10. "Couette flow," Velocity profile for Gr with $M=1, N=1, \omega=$ $1, D a=0.8, R e=1, P e=1, \lambda=1, W e=0.05, \alpha=0.02, \xi=\frac{\pi}{4}, V 0=$ $0.5, t=0.5$.

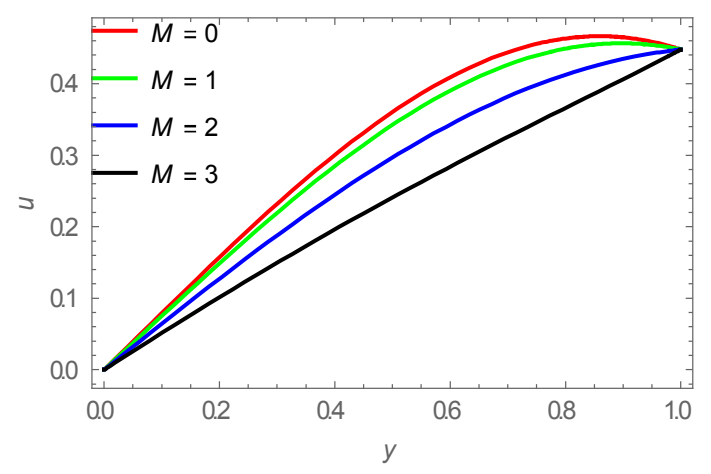

Figure 11. "Couette flow," Velocity profile for $M$ with $G r=1, N=1, \omega=$ $1, D a=0.8, R e=1, P e=1, \lambda=1, W e=0.05, \alpha=0.02, \xi=\frac{\pi}{4}, V 0=$ $0.5, t=0.5$. 


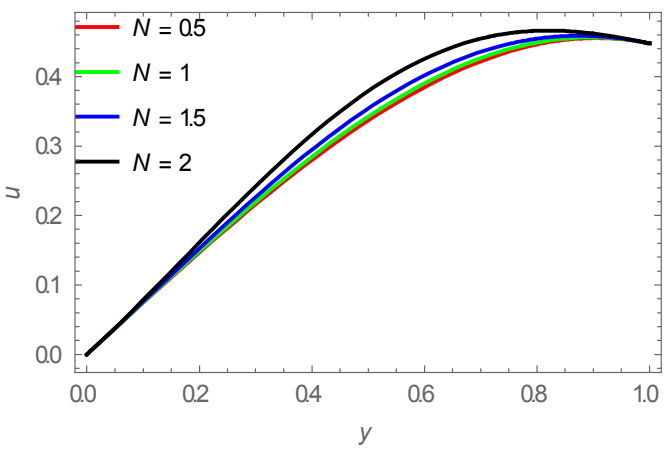

Figure 12. "Couette flow," Velocity profile for $N$ with $\mathrm{Gr}=1, M=1, \omega=$ $1, D a=0.8, R e=1, P e=1, \lambda=1, W e=0.05, \alpha=0.02, \xi=\frac{\pi}{4}, V 0=$ $0.5, t=0.5$.

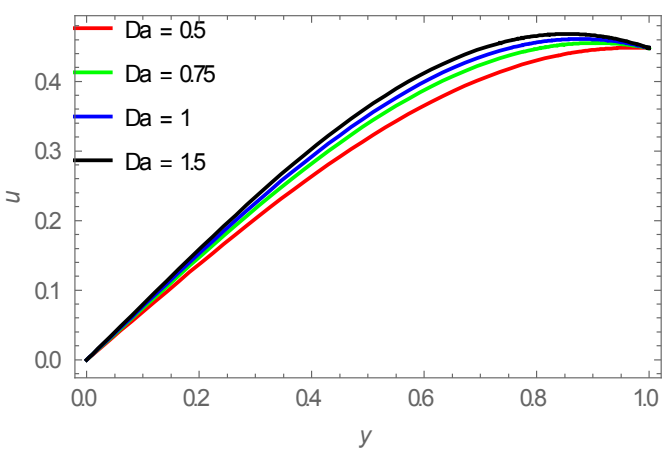

Figure 13. "Couette flow," Velocity profile for Da with $\mathrm{Gr}=1, M=1, \omega=$ $1, N=1, R e=1, P e=1, \lambda=1, W e=0.05, \alpha=0.02, \xi=\frac{\pi}{4}, V 0=$ $0.5, t=0.5$.

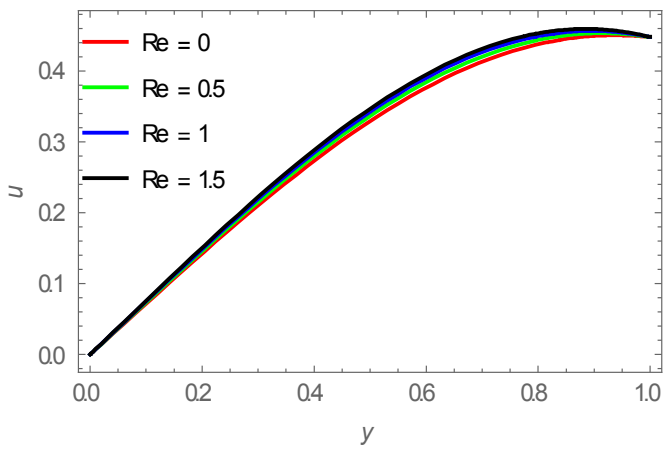

Figure 14. "Couette flow," Velocity profile for Re with $\mathrm{Gr}=1, M=1, \omega=$ $1, N=1, D a=0.8, P e=1, \lambda=1, W e=0.05, \alpha=0.02, \xi=\frac{\pi}{4}, V 0=$ $0.5, t=0.5$.

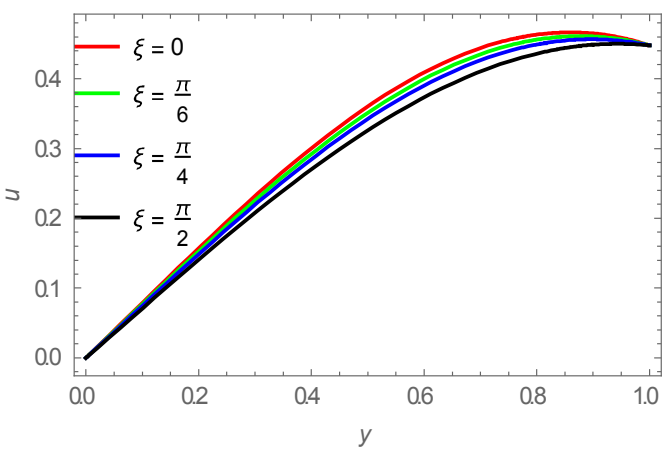

Figure 15. "Couette flow," Velocity profile for $\xi$ with $\mathrm{Gr}=1, M=1, \omega=$ $1, N=1, D a=0.8, R e=1, \lambda=1, W e=0.05, \alpha=0.02, P e=1, V 0=$ $0.5, t=0.5$.

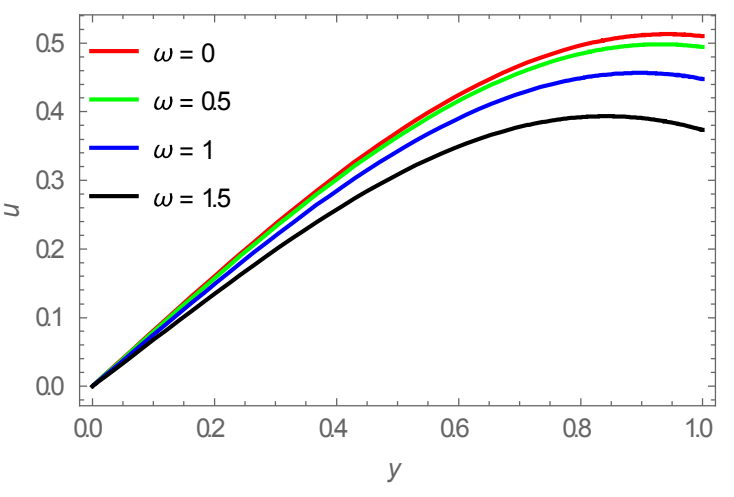

Figure 16. "Couette flow," Velocity profile for $\omega$ with $\mathrm{Gr}=1, M=1, \mathrm{Re}=$ $1, N=1, D a=0.8, R e=1, \lambda=1, W e=0.05, \alpha=0.02, \xi=\frac{\pi}{4}, V 0=$ $0.5, t=0.5$.

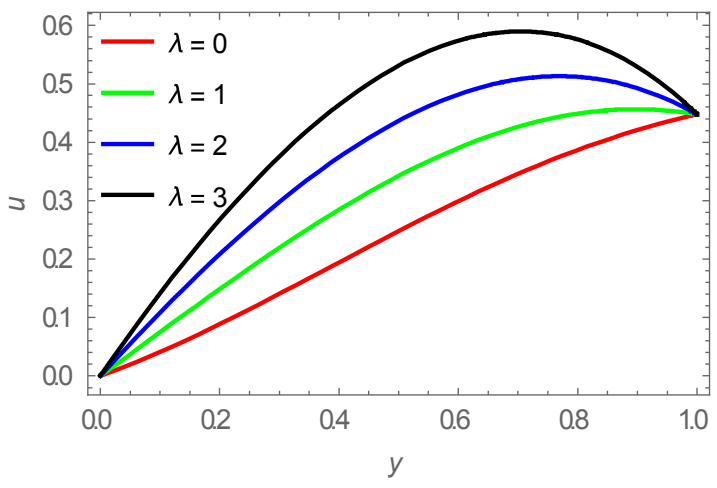

Figure 17. "Couette flow," Velocity profile for $\lambda$ with $\mathrm{Gr}=1, M=1, \mathrm{Re}=$ $1, N=1, D a=0.8, R e=1, \omega=1, W e=0.05, \alpha=0.02, \xi=\frac{\pi}{4}, V 0=$ $0.5, t=0.5$.

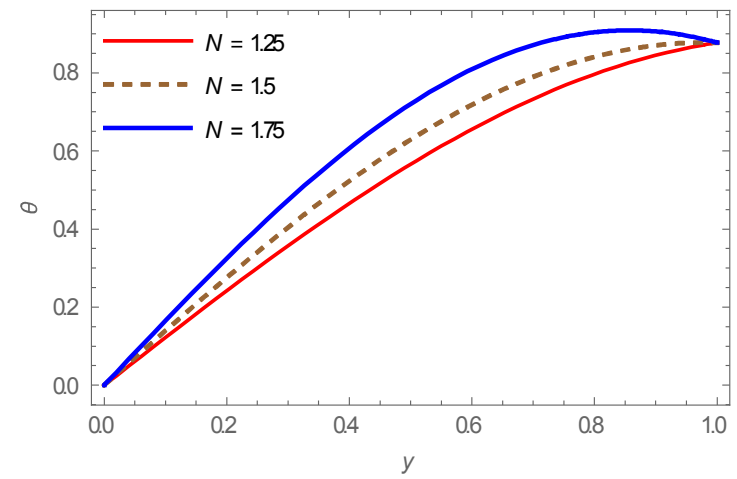

Figure 18. Influence of $N$ on Temperature $\theta$ for $\omega=1, P e=0.7, t=0.5$.

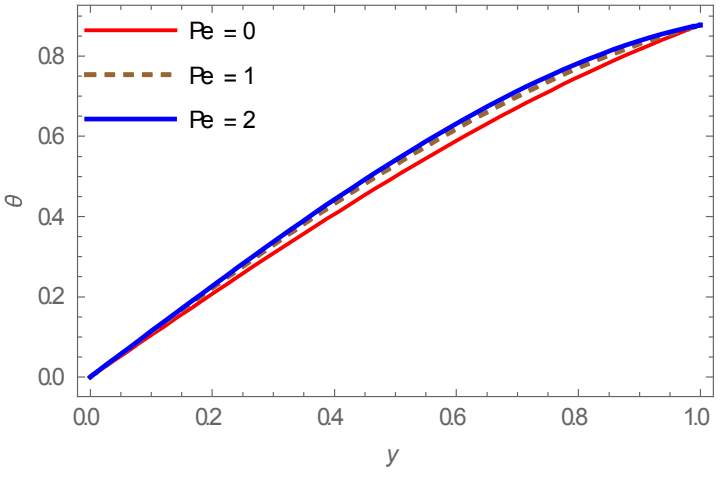

Figure 19. Influence of $P$ e on Temperature $\theta$ for $t=0.5, N=1, \omega=1$. 


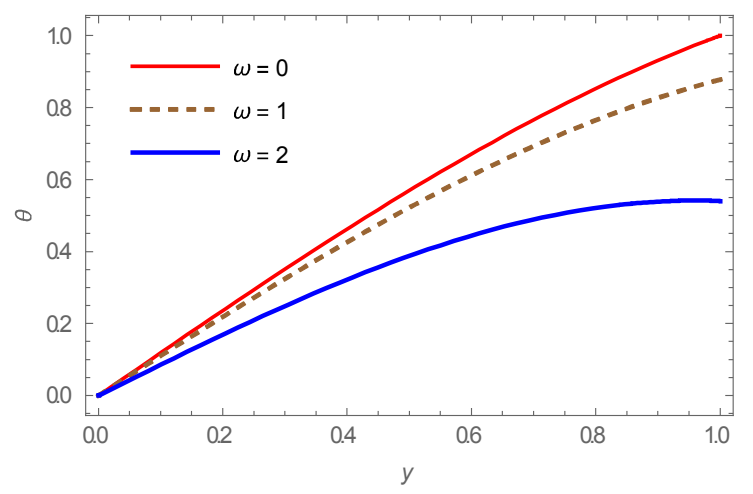

Figure 20. Influence of $\omega$ on Temperature $\theta$ for $t=0.5, N=1, P e=0.7$.

\section{Conclusion and Remarks}

We discuss the influence of heat transfer on MHD oscillatory flow for Williamson fluid with variable viscosity through a porous medium. The velocity and temperature are found analytical, and use different values to find the results of pertinent parameters, namely for the velocity and temperature. The key point is listed below:

i. The velocity profiles increase with increasing radiation parameter $N$, Darcy number $D a$ and Grashof number $\mathrm{Gr}$ for both the Poiseuille and Couette flow.

ii. There are little increases in velocity profiles by increasing Reynolds number $R e$ and $\lambda$ for both the Poiseuille and Couette flow.

iii. The velocity profiles decrease with increasing magnetic parameter $M$, the angle between velocity field and magnetic field strength $\xi$ and frequency of the oscillation parameter $\omega$ for both the Poiseuille and Couette flow.

iv. Show that by the increases radiation parameter $N$ and Peclet number $P e$ the temperature increasing $\theta$ and the temperature $\theta$ decreases by the increasing frequency of the oscillation parameter $\omega$.

\section{References}

[1] T. Hayat, R. Ellahi, F. M. Mahomed, and S. Africa, "Acta Mechanica Exact solutions for Couette and Poiseuille flows for fourth grade fluids," vol. 78, pp. 69-70, 2007.
[2] K. M. Joseph, S. Daniel, and G. M. Joseph, "Unsteady MHD Couette Flow between Two Infinite Parallel Porous Plates in an Inclined Magnetic Field with Heat Transfer," Int. J. Math. Stat. Invent., vol. 2, no. 3, pp. 103-110, 2014.

[3] B. S. D. Nigamf and S. N. Singhj, "Heat Transfer By Laminar Flow Between Parallel Plates Under the Action of transverse magnetic field," Quart. Journ. Mech Applied. Math, vol. XIII, no. 5,1960 .

[4] M. Y. Malik, I. Zehra, and S. Nadeem, "Flows of Carreau fluid with pressure dependent viscosity in a variable porous medium: Application of polymer melt," Alexandria Eng. J., 2014.

[5] I. Zehra, M. M. Yousaf, and S. Nadeem, "Numerical solutions of Williamson fluid with pressure dependent viscosity," Results Phys., vol. 5, pp. 20-25, 2015.

[6] S. Nadeem and M. Awais, "Thin film flow of an unsteady shrinking sheet through porous medium with variable viscosity," Phys. Lett. A, vol. 372, pp. 4965-4972, 2008.

[7] D. G. S. Al-khafajy, "Influence of MHD and Wall Properties on the Peristaltic Transport of a Williamson Fluid with Variable Viscosity Through Porous Medium," Iraqi J. Sci., vol. 58, no. 2, pp. 1076-1089, 2017.

[8] W. S. Khudair and D. G. S. Al-khafajy, Influence of heat transfer on Magneto hydrodynamics oscillatory flow for Williamson fluid through a porous medium, Iraqi J. Sci., vol. 59, no. 1B, pp. 389-397, 2018.

[9] A. A. Khan, R. Ellahi, and K. Vafai, "Peristaltic Transport of a Jeffrey Fluid with Variable Viscosity through a Porous Medium in an Asymmetric Channel," vol. 2012.

[10] T. Hayat, S. Nawaz, A. Alsaedi, and M. Rafiq, "Results in Physics Influence of radial magnetic field on the peristaltic flow of Williamson fluid in a curved complaint walls channel," Results Phys., vol. 7, pp. 982-990, 2017.

[11] S. E. G. A. C. Cogley. W. G. Vincenti, "Differential approximation for radiative transfer in a Nongrey gas near equilbrium," Am. Inst. Aeronaut. Astronaut., vol. 6, no. 3, pp. 551-553, 1968.

[12] J. D. Kevorkian, Jirair, Cole, Perturbation Methods in Applied Mathematics. Springer-Verlag New York, 1981. 